\title{
Autopercepción de calidad de vida: un estudio comparativo
}

Dora Galli *

\section{Resumen}

El concepto Calidad de Vida tiene un componente objetivo, determinado por las condiciones socioeconómicas, y un componente subjetivo que es la autopercepción del bienestar individual. La psicoeducación ofrece recursos para mejorar la calidad de vida de las personas modificando la percepción y el afrontamiento de las condiciones externas. Se investigó si la calidad de vida subjetiva se relaciona con los niveles de calidad de vida objetiva en tres ciudades de la República Argentina. Objetivos: determinar el nivel de calidad de vida objetiva y examinar diferencias individuales de autopercepción de calidad de vida en Capital Federal y las ciudades de Balcarce y Lincoln. Muestra: probabilística, 398 adultos (200 hombres y 198 mujeres). Métodos: a) relevamiento de indicadores sociodemográficos b) administración del test Indice de Calidad de Vida (QLI-Sp). Resultados: los parámetros de calidad de vida objetiva no son determinantes en la autopercepción de bienestar individual.

Palabras clave: Calidad de vida objetiva - Calidad de Vida subjetiva - Psicoeducación

\section{Abstract}

The idea of Quality of Life includes two areas: objective aspects such as socioeconomic status, and subjective self- perception. Psychoeducation provides useful means to find alternative ways to evaluate and cope with life conditions, thus improving quality of life. Evidence to support if there is a relationship between quality of life selfperception and external life conditions was examined in three cities of Argentina. Objective: to assess the socioeconomic status and to analyze individual differences in self-perception among residents of the cities of Buenos Aires, Balcarce and Lincoln. Participants: a total of 398 adults (200 men and 198 women). Method: a) assessment of socioeconomic conditions

according to international standards b) assessment of quality of life self-perception applying the Quality of Life Index (QLI-Sp). Results: external life conditions do not predict individual self- perception of quality of life.

Key Words: Objective Quality of Life - Subjective Quality of Life -Psychoeducation

* Licenciada en Psicología, Universidad de Palermo; psicóloga concurrente del Hospital Francés, Buenos Aires. e-mail: doragalli@yahoo.com.ar. 


\section{Introducción}

El presente estudio surge de la práctica profesional en el Hospital Francés de la Ciudad de Buenos Aires. El Servicio de Psiquiatría de dicha Institución desarrolla investigaciones en el área de Trastornos de Ansiedad y de Estado de Animo, así como también en la Unidad Coronaria. Se realizan mediciones del nivel de calidad de vida autopercibida entre la población psiquiátrica y clínica con afecciones cardiovasculares, administrando el instrumento denominado Indice de Calidad de Vida. El marco teórico corresponde al modelo cognitivo, con orientación constructivista realista.

El tema seleccionado se refiere a la autopercepción de calidad de vida entre población general en ciudades con diferentes niveles de calidad de vida objetiva

\section{Psicología de la Salud: antecedentes históricos y evolución}

Tradicionalmente la psicología se ha ocupado más del estudio de temas relacionados con la patología, la enfermedad y los desajustes, de aquello que tiene que ver con los síntomas clínicos. (Veenhoven, 1988, 1991). A lo largo de años se descuidó el análisis del efecto que el bienestar y la felicidad tenían sobre el funcionamiento psicológico personal y otros aspectos generadores salugénicos (Ryff y Keyes, 1995).

Desde hace algunos años se ha incrementado la difusión de la Psicología Positiva, que postula un enfoque salugénico, trabajando en la prevención de enfermedades y promoción de la salud. Dicho enfoque puede aplicarse a intervenciones en el ámbito individual así como incluirse en programas y políticas a nivel comunitario.

La Psicología la Salud entendida como "la rama aplicada de la Psicología que se dedica al estudio de los componentes subjetivos y de comportamiento del proceso de salud-enfermedad y de la atención de salud" (Calatayud, 1999), es una vertiente cuyos campos involucran una multiplicidad de temáticas al incorporar el estudio del componente subjetivo tanto en las enfermedades de manifestación orgánica como en las de manifestación mental, sus determinantes, factores protectores, y las acciones que se efectúen para contrarrestarlas en las actividades de promoción, prevención, recuperación y rehabilitación.

En relación a la promoción de la salud y a lo que atañe a la generación de desarrollos teóricos y técnicos en torno a la salud positiva, será esencial rescatar el concepto de salutogénesis (Antonovski, 1979,1987) en donde la pregunta no pasa por establecer cuál es la causa de la enfermedad sino por conocer cuáles son los factores que hacen que ciertos individuos de una determinada población no adquieran ésta o aquella enfermedad. Desde esta perspectiva es que se pueden realizar conceptualizaciones sobre factores protectores, resiliencia, sentido de coherencia o procesos de cambio o acciones que realizan las personas para mantener su salud. También indagar cuáles son los factores del ambiente humano que dan la condición de posibilidad para que esto ocurra, sin desconocer el nivel de lo general, indagar el nivel de lo particular.(Samaniego, 2002) 


\section{Calidad de Vida}

En un primer momento la expresión Calidad de Vida aparece en los debates públicos en torno al medio ambiente y al deterioro de las condiciones de vida urbana. Durante la década de los 50 y a comienzos de los 60 , el creciente interés por conocer el bienestar humano y la preocupación por las consecuencias de la industrialización de la sociedad hacen surgir la necesidad de medir esta realidad a través de datos objetivos, y desde las Ciencias Sociales se inicia el desarrollo de los indicadores sociales, estadísticos que permiten medir datos y hechos vinculados al bienestar social de una población. Estos indicadores tuvieron sus propia evolución siendo en un primer momento referencia de las condiciones objetivas, de tipo económico y social, para en un segundo momento contemplar elementos subjetivos. (Arostegui, 1998).

Transcurridos 20 años, aún existe una falta de consenso sobre la definición del constructo y su evaluación. Así, aunque históricamente han existido dos aproximaciones básicas: aquella que lo concibe como una entidad unitaria, y la que lo considera un constructo compuesto por una serie de dominios, todavía en 1995, Felce y Perry encontraron diversos modelos conceptuales de Calidad de Vida.

A pesar de esta aparente falta de acuerdo entre los investigadores sobre la definición de calidad de vida y la metodología utilizada para su estudio, el concepto ha tenido un impacto significativo en la evaluación y planificación de servicios durante los últimos años.

En líneas generales, para Schalock (1996) la investigación sobre Calidad de Vida es importante porque el concepto está emergiendo como un principio organizador que puede ser aplicable para la mejora de una sociedad como la nuestra, sometida a transformaciones políticas, sociales, tecnológicas y económicas. No obstante la verdadera utilidad del concepto se percibe sobre todo en los servicios humanos, inmersos en una llamada Quality Revolution que propugna la planificación centrada en la persona y la adopción de un modelo de apoyos y de técnicas de mejora de la calidad.

En este sentido, el concepto puede ser utilizado para una serie de propósitos, incluyendo la evaluación de las necesidades de las personas y sus niveles de satisfacción; la evaluación de los resultados de los programas y servicios humanos; la dirección y guía en la provisión de estos servicios, y la formulación de políticas nacionales e internacionales dirigidas a la población general y a otras más específicas. (Gómez-Vela, Sabeh, 2000)

Según Saforcada (1998), como es sabido se han logrado avances debido al control de los agentes biológicos que causan las enfermedades infectocontagiosos y en los recursos médicos para curarlas. Así, las enfermedades crónicas y las lesiones ocupan ahora los primeros lugares en las causas de muerte y morbilidad en muchos países, fundamentalmente en aquellos con mayor desarrollo y en los núcleos de población urbana de casi todos. Estos problemas tanto en su origen como en su evolución están notablemente asociados al modo de vida, las condiciones de vida y trabajo, las tensiones de la vida, las relaciones en los grupos primarios y en la sociedad en general, y al llamado estilo de vida individual. 
Otro cambio que se relaciona con el anterior es el que se viene operando en el pensamiento salubrista que es cualitativamente diferente a la posición clínica. Ofrece las herramientas necesarias para la correcta interpretación de los problemas de salud de las poblaciones y para orientar las soluciones. Al reconocer la multicausalidad de la salud y la enfermedad, esta posición destaca la subordinación de lo biológico a lo social y el papel mediatizador de la personalidad del ser humano, sujeto activo en su relación con la realidad. (Saforcada, 1998)

Cada día hay más expansión de un modo de hacer psicología que aborda progresivamente la prevención y promoción de la salud con mayor frecuencia.

La caracterización psicosocial del concepto de Calidad de Vida y su relación con la salud permite ver que dicha calidad no está inevitablemente atada las condiciones económicas, si éstas no imposibilitan la satisfacción de las necesidades esenciales de la vida. La condición dificultosa en el plano económico no es impedimento insalvable para lograr una mejor calidad de vida o para evitar que ésta se degrade.

De un escenario de salud colectiva en el que tenían la mayor importancia los factores biológicos, se ha pasado a otro en el que la investigación científica y las prácticas profesionales muestran que los factores con mayor poder de determinación, en el proceso de salud-enfermedad, son de naturaleza psicosociocultural. Esto lleva a pensar que la marejada de la crisis económica que acosa a América Latina no nos debe impedir la comprensión de que la calidad de vida de nuestros pueblos depende esencialmente de los valores compartidos, de las actitudes que permitan mancomunar los esfuerzos y generar procesos de adaptación y aceptación de los unos hacia los otros, lo que traerá aparejado un mejoramiento de los indicadores de salud y una mejor condición para afrontar la crisis y las causas determinantes de ella. (Saforcada,1998).

Según Samaniego (2002), cuando hablamos de vulnerabilidad psicosocial estamos acentuando aquellas características propias de un sujeto o grupo de sujetos que hacen que ellos mismos tengan una probabilidad mayor de padecer un daño. Es decir que, sin dejar de tener en cuenta los factores de riesgo ubicables en el ambiente, en este punto el foco de atención está puesto sobre aspectos clasificados como factores de riesgo endógenos en la epidemiología clásica: variables psicosociales y su relación con la vulnerabilidad. Lo más ampliamente trabajado en este sentido, es su relación con el concepto estilos de vida. En muchos estudios en los países desarrollados el concepto de estilo de vida aparece asimilado al de conductas o hábitos individuales, y según cuales adopten los sujetos se incrementará o no su riesgo de padecer distinto tipo de afecciones.

Se estima que alrededor del $42 \%$ de las patologías se relacionan con los estilos de vida, mientras que el resto se distribuye entre las variables de medio ambiente, servicios de salud y biología humana.

Asumiendo que los estilos de vida se materializarán en conductas concretas, conceptualizaremos a éstas como la expresión de la personalidad mediatizada por sistemas reguladores internos. La personalidad no es un reservorio interno de respuestas prefabricadas con comportamientos idóneos para cada situación externa que afrontamos, 
ni es ella la que enfrenta la situación: quien lo hace es el hombre como sujeto de su sistema personalidad. (Samaniego, 2002)

Muchos autores se han ocupado a lo largo de la historia, de estudiar en qué consiste "vivir y sentirse bien”. ¿Por qué algunas personas se sienten mejor que otras? ¿Por qué algunos están más satisfechos con su vida? ¿Cuáles son las causas de la insatisfacción? Estas preguntas han servido de guía a los estudios actuales sobre la satisfacción vital y el bienestar psicológico. Durante años se creyó que sentirse satisfecho con la vida estaba muy relacionado con la inteligencia, la apariencia física o las buenas condiciones económicas. Hoy en día se sabe que el mayor o menor bienestar psicológico no está relacionado sólo con variables desde una perspectiva macroscópica: indicadores económicos de una sociedad, la desigualdad social, la existencia de situaciones de pobreza y marginalidad, el respeto por los derechos humanos, conflictos sociales o desempleo crónico, sino también con la subjetividad individual.(Casullo et al, 2002)

¿Qué contexto favorece a una mejor calidad de vida? ¿Las grandes ciudades o las pequeñas aldeas rurales? Desde la sociología se han caracterizado los factores que inciden sobre la calidad de vida y el bienestar en las ciudades: alta densidad poblacional, aislamiento y desorganización (Adams, 1992). Las consecuencias psicológicas de estos factores resultan claras: los ámbitos más urbanizados traen consecuencias negativas para las relaciones sociales y la salud mental. (Sarason, 1974). El modelo sistémico sostiene que, al ser los ámbitos más estables, tal el caso de las pequeñas ciudades, la población registra menores cambios, se incrementan las redes sociales y por lo tanto el bienestar psicológico y la calidad de vida aumentan (Fischer, 1973). Por otro lado, el modelo de desarrollo lineal predice que en tanto el tamaño y la diversidad de una población aumentan, las personas se vuelven más individualistas, egoístas y aisladas de los demás; por ende la satisfacción vital para esa población disminuye, y las personas reconocen sentimientos de extrañamiento, aislamiento y ausencia de control sobre sus vidas (Milgram, 1970).

Existen muchas opiniones, teorías y modelos, pero pocas contrastaciones empíricas. Se han localizado muy pocos estudios que hayan verificado empíricamente las cuestiones mencionadas. Se encontraron estudios con resultados incongruentes.

Para Adams (1992) la cuestión es mucho más difícil que establecer simplemente si los ámbitos urbanos promueven o no más bienestar que los rurales, ya que intervienen variables moderadoras diversas. La variable que más diferencia ambos entornos es la densidad poblacional: a mayor cantidad de personas extrañas en el vecindario próximo decrece la expectativa de control sobre el medio y por lo tanto existe menor nivel de bienestar psicológico. En la misma línea están los hallazgos de Saber, Shenen y Boymel (1976), quienes encontraron que no existe relación entre lugar de residencia y satisfacción. Kishman y Lane (1980) no hallaron diferencias significativas entre residentes de ámbitos urbanos y rurales de una muestra de adultos del estado de California.

Para Pagliccia, Apland y Kazankian (1995) el impacto del tamaño de las ciudades debe analizarse desde la experiencia de urbanización percibida por los propios actores sociales, pues es más importante que los indicadores objetivos (Casullo et al, 2002). 


\section{Importancia de la Evaluación Cognitiva y el Afrontamiento de la situaciones en el manejo del stress}

Las tradiciones culturales han cumplido la función histórica de dar significado y orden a la vida social, y de guiar a los miembros del grupo con un sistema de valores, creencias y objetivos compartidos. Estas tradiciones, trasmitidas de padres a hijos, proporcionan al niño un anteproyecto que le permite organizar sus pensamientos, comportamientos y aspiraciones. Uno de los problemas con que nos encontramos hoy en día es el cambio social y las normas cada vez más contradictorias que los miembros de nuestra sociedad tienen que suscribir. No podemos encontrar certezas ni valores. La complejidad y la diversidad de la experiencia diaria causan estragos en las creencias simples arcaicas y las vuelven inútiles como instrumentos para manejar las realidades actuales. (Millon, 1998).

La sociedad y la cultura redujeron pendularmente las prohibiciones y mandatos restrictivos patológicos por su exceso, hasta el límite de un equívoco laissez faire. El nuevo paradigma de la educación es complaciente con los deseos de cada individuo. Olvidamos que aquella represión, por cierto excesiva, en su justa medida servía para ordenar la convivencia al imponer límites, justificados por valores compartidos, lo que hoy llamamos el capital social. Su carencia genera conductas conflictivas entre la gente, generando una patología prevalente: la violencia. La sobreadaptación de antes se convirtió en inadaptación y la violencia está en la relación entre las personas, en la sociedad. Como la relación entre los individuos pertenece al ámbito de lo social, se hablaría entonces del concepto de salud social: conflictos conyugales o entre padres e hijos, conductas violentas hacia los demás o hacia uno mismo, en los abusos de toda índole y en las conductas criminales. (Usandivaras, 2004).

¿Qué condiciones objetivas y subjetivas hacen que el bienestar psicológico se eleve, disminuya o mantenga? ¿Cómo incide en la calidad de vida de las personas? ¿Puede elevarse o mejorarse mediante técnicas específicas de intervención? Taylor y Brown (1989) destacan la importancia de las "ilusiones positivas" como criterio de salud mental, es decir, considerar que el presente y el futuro pueden ser mejores, sin importar las condiciones objetivas que impone la realidad. .(Casullo, et al, 2002).

Según Belloch, Sandín y Ramos (1995), actualmente el stress constituye un considerable factor de riesgo de patologías orgánicas y psicológicas. Los estímulos que provocan stress o estresores pueden provenir del medio externo, como condiciones de vida desfavorables, lo cual disminuye la calidad de vida objetiva, o del medio interno, como la evaluación cognitiva incorrecta de situaciones y de los propios recursos así como estrategias de afrontamiento desadaptativas, con incidencia negativa en la autopercepción de la calidad de vida, o calidad de vida subjetiva.

Los sucesos vitales y los acontecimientos diarios ejercen demandas sobre el organismo que son valoradas por éste. Dependiendo de cómo sea esta valoración se producirá o no la respuesta de estrés. Esta ocurrirá únicamente si se produce ruptura del balance entre la percepción de la demanda y la percepción de los recursos personales 
para superarla. (Cox, 1978). Es decir, si la persona percibe que el suceso supera o agrava sus recursos -afrontamiento, apoyo social, etc. (Lazarus \& Folkman, 1984). Se vio la necesidad de incluir variables de corte cognitivo -evaluación, percepción, etc.- es decir, ubicadas en la misma persona.

Dependiendo de la forma en que el sujeto evalúe ciertas características del estresor como la deseabilidad, controlabilidad, negatividad, predecibilidad, novedad y dependiente versus independiente, puede variar el efecto del suceso vital. Cuando el sujeto percibe los sucesos como negativos, incontrolables, impredecibles, independientes e indeseables, por ejemplo, suelen perturbar la salud de forma más prominente. (Belloch et al, 1995)

Debemos puntualizar que la valencia de estas dimensiones no siempre está determinada únicamente por la valoración o percepción que hace la persona. También el tipo de situación puede ser hasta cierto punto determinante. Existe más bien una interacción entre ambas, siendo menos manifiesta la influencia de la percepción/valoración de la persona cuando la imposición ambiental es mayor, y viceversa.

Las estrategias de afrontamiento - coping- se refieren a los esfuerzos conductuales y cognitivos que emplea el sujeto para hacer frente a las demandas concretas valoradas como gravamen, así como también para suprimir el estado emocional del estrés.

El apoyo social se ha referido también como un factor importante asociado al estrés. El mismo puede amortiguar el efecto de las demandas estresantes sobre las respuestas de estrés. También parece que puede actuar directamente sobre la salud. El estatus de salud, tanto psicológico como fisiológico, así se trata de un resultado del proceso de estrés. Un afrontamiento inapropiado puede ser nocivo para la salud. (Belloch et al, 1995).

Las terapias de estilo cognitivo se centran en cómo perciben los pacientes los acontecimientos de sus vidas, basándose en cómo procesan la información, organizan sus pensamientos y comunican sus reacciones e ideas a los demás. Estas terapias proporcionan uno de los índices más útiles para el clínico, refiriéndose a la distinta manera de funcionar del paciente. Sintetizando estos datos, es posible identificar características generales como el pensamiento constreñido, la distractibilidad cognitiva, el pensamiento empobrecido. Se neutralizan los pensamientos erróneos o distorsionados, cualquiera que sea su temática. Las distorsiones de la percepción y las distorsiones cognitivas pueden considerarse el lado defectivo de un proceso normal en el que los nuevos estímulos se consideran similares a los experimentados en el pasado. Se suele hacer referencia a este proceso como generalización de estímulos. La tendencia a generalizar comportamientos inadecuados tiene consecuencias en especial a largo alcance, porque suelan agravar las condiciones que las hacen surgir.(Millon, 1998).

Un problema es una situación en la que se intenta alcanzar un objetivo y se hace necesario encontrar un medio para conseguirlo. Este objetivo no se puede alcanzar con el repertorio comportamental actual del sujeto: éste debe crear nuevas acciones e integraciones. Se han planteado estrategias específicas para proporcionar herramientas que ayuden a la solución creativa de problemas. No solamente se busca lograr que la persona represente de forma realista el problema, sino en algunos casos 
lograr una redefinición del problema de manera que presente posibilidades de solución.(Bulacio, 2004)

\section{La Psicoeducación como estrategia para elevar la Calidad de Vida}

La teoría constructivista realista de la psicología cognitiva postula que la psicoeducación es una herramienta fundamental para enseñar a las personas a optimizar los recursos con los que cuenta, tanto externos como internos, a través de una evaluación cognitiva que les permita elaborar estrategias de afrontamiento y participar activamente en la construcción de una realidad más favorable para sí mismo y su entorno.

De acuerdo con Bulacio (2004), la psicoeducación es un proceso que permite brindar a las personas la posibilidad de desarrollar y fortalecer capacidades para afrontar las diversas situaciones de un modo más adaptativo, posibilitando mayor flexibilidad y realismo en el modo de abordar la problemática y su contexto. La psicoeducación por lo tanto es un proceso amplio que puede orientarse a la psicopatología o a personas en general, y su intencionalidad es mejorar la calidad de vida acorde al bien común. La psicoeducación debe ayudar a dar significados nuevos a las experiencias, generando nuevas estrategias psicológicas de afrontamiento. La calidad de vida depende en gran medida de la valoración personal que realiza el individuo, son los aspectos subjetivos de la calidad de vida los que toman relevancia,.

En la sexta revisión de la Clasificación Internacional de Enfermedades -CIE6-, por la Organización Mundial de la Salud, comienza a incluirse poco a poco el concepto de bienestar frente al de enfermedad, enfatizándose los aspectos positivos de salud en consideración del estado salud-enfermedad de una persona. Conceptualmente se puede hacer eje en condiciones objetivas o en la percepción subjetiva del individuo, integrándose la calidad de vida como la combinación de componentes objetivos y subjetivos-condiciones de vida más satisfacción personal- ponderados por la escala de valores, aspiraciones y expectativas personales.

Creemos que las distorsiones colectivas pueden aunque sea parcialmente modificarse desde un compromiso con la psicoeducación para contribuir a un hombre más humano o sea con criterios propios, en definitiva, más libre y responsable. (Bulacio, 2004)

Podría considerarse que la calidad de vida tiene un componente objetivo, determinado por variables como el contexto socioeconómico, el estado de salud y el nivel educativo, y un componente subjetivo individual, que es la autopercepción de la calidad de vida.

En base a la hipótesis que la calidad de vida autopercibida no está relacionada con los parámetros de calidad de vida objetiva, el presente estudio intenta explorar los efectos que las diferentes condiciones de vida en Capital Federal y en dos ciudades de la provincia de Buenos Aires con distintos niveles de calidad de vida objetiva tienen sobre la autopercepción de calidad de vida de sus habitantes.

Cuando las condiciones socioeconómicas disminuyen considerablemente los niveles de calidad de vida objetiva de un individuo o población, como ocurre en la Argentina, cobraría especial relevancia investigar el grado de relación de ambos componentes. 
Según cifras difundidas en octubre del 2004 por el Instituto Nacional de Estadísticas y Censos -INDEC-, el 44,3\% de la población argentina es pobre. Esto implica que actualmente sobre la población total, existen 16 millones de pobres en todo el país. Los hogares por debajo de la línea de indigencia llegaron en el primer semestre del año en curso al $17 \%$, indicando que unas 6.300 .000 personas son indigentes.

En virtud de lo precedente, se considera relevante considerar la posibilidad de mitigar los efectos negativos que sobre la calidad de vida total de una persona provoca la disminución de la calidad de vida objetiva. Dicho objetivo podría llevarse a cabo elevando los niveles de calidad de vida subjetiva a través de la implementación de programas psicoeducativos aplicables en ámbitos clínicos y comunitarios.

¿Existen diferencias de calidad de vida subjetiva entre ciudades con distintos niveles de calidad de vida objetiva? ¿El tamaño y la densidad de una población inciden sobre la calidad de vida?

Los objetivos de la presente investigación son:

1- Determinar la calidad de vida objetiva de las ciudades participantes: Capital Federal-Balcarce-Lincoln, según parámetros de la Organización Panamericana de Salud.

2- Examinar las. diferencias individuales en la calidad de vida autopercibida de habitantes según el lugar de residencia, Capital Federal- Balcarce-Lincoln.

3- Establecer diferencias individuales según sexo y edad.

\section{Método}

\section{Muestra}

La muestra en las tres ciudades es de tipo probabilística, y consiste de población general no consultante, edad mínima 18 años, sin límite máximo de edad, ambos sexos.

Capital Federal: La muestra quedó constituida por $n=300$. La media de edad es de 47.50 y el desvío típico (DT) es de 18,5.

Balcarce: La muestra estuvo compuesta por 50 sujetos, cuya media de edad es de 41.78 y el desvío típico (DT) es de 15,8.

Lincoln: La muestra consta de 48 individuos, siendo la media de edad de 40,27 y el desvío típico de 16,4.

\section{Instrumentos}

\section{Medición de indicadores de calidad de vida objetiva:}

Se incluyen datos sobre la calidad de vida objetiva, o sea condiciones externas relacionadas a: salud, estándar de vida, nivel educacional, longevidad, empleo, seguridad pública, condiciones de vivienda e indicadores demográficos de la Capital Federal, las ciudades de Balcarce y Lincoln. Estos datos fueron recogidos por el INDEC y los 
gobiernos provinciales y municipales de acuerdo a estándares de la Organización Panamericana de la Salud, Organización Mundial de la Salud, y el Programa de las Naciones Unidas para el Desarrollo.

\section{Medición de calidad de vida subjetiva}

El Índice de Calidad de Vida-QLI-Sp- incluye 10 ítems, con formato de respuesta de opción múltiple Likert, compuesto por una escala que va de 1 a 10. Estos ítems representan, según los autores, diez aspectos relevantes para la evaluación del constructo calidad de vida:

1. bienestar físico,

2. bienestar psicológico-emocional

3. cuidado personal y funcionamiento independiente,

4. funcionamiento ocupacional,

5. funcionamiento interpersonal,

6. apoyo emocional y social,

7. apoyo estatal y de servicios,

8. plenitud personal,

9. plenitud espiritual

10. percepción global de la calidad de vida

Cada ítem es valorado por el sujeto según su perspectiva personal en el momento actual.

Este instrumento fue diseñado originalmente en cuatro idiomas: inglés, español, chino y coreano y presenta índices de aplicabilidad, fiabilidad y validez adecuados.

Bulacio, Vieyra, Daneri, Benatuil \& Mongiello, (2004) han adaptado y validado dicho instrumento para la Argentina en su versión española-QLI-Sp-. Para ello se ha solicitado la autorización de los autores Mezzich, Cohen, Ruipérez, Yoon, Liu.

\section{Resultados}

\section{Calidad de Vida objetiva:}

Se han revisado 13 variables de diversa índole aceptadas por la Organización Panamericana de la Salud y el Programa de las Naciones Unidas para el Desarrollo OPS y PNUD- como indicadores válidos de calidad de vida objetiva: densidad demográfica, analfabetismo, tipo de vivienda, servicio sanitario de la vivienda, calidad de materiales de la vivienda, nivel educacional, tasa de desocupación, necesidades básicas insatisfechas, cobertura de salud, longevidad, tasa de mortalidad materna, tasa de mortalidad ajustada por edad y tasa de mortalidad infantil.

La comparación arroja resultados a favor de la ciudad autónoma de Buenos Aires en la totalidad de los mencionados indicadores de calidad de vida objetiva, con excepción de la variable densidad demográfica. 
Las variables medidas en forma directa en las ciudades de Balcarce y Lincoln son cinco: densidad demográfica, analfabetismo, tipo de vivienda, servicio sanitario de la vivienda y calidad de materiales de la vivienda. La comparación de calidad de vida objetiva entre ambas localidades se realizó en base a dichas variables.

Se observan leves diferencias a favor de la ciudad de Lincoln, salvo en los indicadores de servicios sanitarios de las viviendas.

En síntesis, de acuerdo a los datos relevados precedentemente el nivel de calidad de vida objetiva de las ciudades de Balcarce y Lincoln sería inferior al de la Capital Federal. Por otro lado, dicho nivel no difiere significativamente entre ambas localidades provinciales.

La tasa de analfabetismo en la ciudad de Balcarce es alrededor de un 14,7\% más elevada que en la ciudad de Lincoln. Ambos valores son respectivamente 4,8 y 4,2 veces superiores respecto de los indicadores de la Capital Federal. La densidad urbana por $\mathrm{km}^{2}$ en la Capital Federal es aproximadamente un 3,5 veces mayor que en las otras dos ciudades de la Provincia de Buenos Aires, cuyos indicadores no difieren significativamente entre sí.

El 93,58\% de la población de la Capital Federal habita en mejores condiciones habitacionales en cuanto a provisión de agua corriente, red cloacal, inodoros, etc. Esta proporción se reduce a $81,96 \%$ y 83,85 en las ciudades de Balcarce y Lincoln respectivamente., aumentando en estas dos localidades considerablemente la población que habita en condiciones precarias - casa B, ranchos y casillas. Los indicadores de ambas ciudades en estas categorías no difieren significativamente entre sí.

Las condiciones de habitabilidad, en este caso con respecto a los materiales de las viviendas, es superior en la Capital Federal, y decrece en forma pareja para las otras dos ciudades objeto de este estudio.

Con respecto a los niveles de población desocupada, los indicadores arrojan una diferencia de 5,3\% a favor de la Capital Federal.

El índice de nivel educacional es significativamente superior en la población de la Capital Federal. La brecha entre Capital Federal y la Provincia de Buenos Aires cobra mayor amplitud en los porcentajes de personas con universitario completo $-8,76 \%$ y secundario completo - 8,53\%, a favor de Capital Federal. Es notable la deserción educativa en la Provincia de Buenos Aires, siendo bajo el número de población que accede y completa niveles secundarios, terciarios y universitarios de educación.

Los datos expresan diferencias a favor de la Capital Federal, que se pronuncian en los indicadores del nivel de longevidad, 6,5\% y la tasa de mortalidad infantil, 5,8\%. Asimismo los índices de cobertura de salud privada y obra social señalan una mejor situación sanitaria en la Capital Federal, cuyos indicadores son un 12,6\% inferiores a los valores de la provincia de Buenos Aires. Las necesidades básicas insatisfechas NBI-, son 2 veces mayor en la provincia de Buenos Aires. La tasa de mortalidad materna en la provincia de Buenos Aires es 2,28 veces mayor que entre la población de Capital Federal. 


\section{Calidad de Vida subjetiva:}

\section{Comparación de las medias según edad}

Se han agrupado las edades de los participantes de las tres muestras $(n=398)$ en cuatro grupos etáreos:

1. 18-32 años;

2. 33-47 años;

3. 48-62 años;

4. 63 años en adelante.

No se han encontrado diferencias significativas entre los grupos en cuanto a la comparación de las medias de cada ítem que compone el Indice de Calidad de Vida, con una excepción.

El análisis arrojó diferencias considerables entre los grupos etáreos 1 (18-32 años) 4 (63 años en adelante), en dos de los ítems: Bienestar Físico (ítem 1) y Apoyo Estatal y de Servicios (ítem 7). A continuación se presentan detalles de las diferencias.

Tabla 2. Comparación de las medias por edades, rangos etéreos 1 y 4.

\begin{tabular}{lcccccc}
\hline & \multicolumn{2}{c}{$\mathbf{1 8 - 3 2}$ años } & \multicolumn{3}{c}{$\mathbf{6 3}$ años y más } \\
& media & ds & media & ds & t \\
\hline Bienestarfísico & 7,34 & 1,62 & 6,74 & 2,06 & $1,65^{*}$ \\
\hline Apoyo Estatal y de Svcios. & 6,78 & 2,07 & 6,14 & 2,46 & $2,05^{*}$ \\
\hline
\end{tabular}

$* p<0,05$

Las personas mayores de 63 años expresan niveles menores de calidad de vida con respecto a los ítems Bienestar Físico y Apoyo Estatal y de Servicios que las personas comprendidas entre los 18 y 32 años.

\section{Comparaciones por sexo en BuenosAires-Balcarce-Lincoln}

No se observan diferencias significativas en el puntaje del item 10 (Percepción Global de la Calidad de Vida) tanto en la ciudad de Buenos Aires como Balcarce entre hombres y mujeres. Esto significa que ambos sexos dicen percibir grados similares de satisfacción y felicidad con su vida en general.

En la ciudad de Lincoln se han encontrado diferencias significativas entre ambos sexos en cuanto a la percepción global de su calidad de vida, arrojando valores más elevados en las mujeres. 
Tabla 3. Comparación por sexo. Percepción Global de Calidad de Vida BsAs-Balcarce-Lincoln

\begin{tabular}{llccccc}
\hline & & $\mathbf{n}$ & media & desv. típ. & t & \\
\hline \multirow{2}{*}{ Buenos Aires } & mujeres & 141 & 7,47 & 1,57 & & \\
& hombres & 159 & 7,31 & 1,77 & 0,79 & NS \\
\hline \multirow{2}{*}{ Balcarce } & mujeres & 28 & 8,00 & 1,12 & & \\
& hombres & 22 & 8,05 & 1,53 & $-0,12$ & NS \\
\hline \multirow{2}{*}{ Lincoln } & mujeres & 29 & 8,21 & 0,98 & & \\
& hombres & 19 & 7,21 & 2,23 & 2,13 & $*$ \\
\hline
\end{tabular}

$* p<0,05$

Comparacionesen la Percepción Global de la Calidad de Vida entre ciudades.

\section{Muestras de las ciudades de Buenos Aires y Balcarce}

Se comparan a continuación los resultados de las puntuaciones del item 10 . (Percepción Global de la Calidad de Vida) y del promedio de los 10 items del instrumento (promedio de la Calidad de Vida), entre las muestras obtenidas en las ciudades de Buenos Aires y Balcarce. Se observan diferencias estadísticamente muy significativas a favor de Balcarce. Esto permite afirmar que la población relevada en dicha ciudad $(n=50)$ autopercibe un nivel más elevado de calidad de vida que la población $(n=300)$ de Buenos Aires (según tabla 16).

Tabla 4. Comparación de Calidad de Vida - Buenos Aires-Balcarce

\begin{tabular}{lrcccc}
\hline & n & media & desv. típ. & t & \\
\hline Percepción global Buenos Aires & 300 & 7,39 & 1,68 & & \\
Balcarce & 50 & 8,02 & 1,30 & $-2,55$ & $* *$ \\
\hline Promedio CV Buenos Aires & 300 & 7,40 & 1,14 & & \\
Balcarce & 50 & 7,96 & 1,67 & 2,99 & $* *$ \\
\hline
\end{tabular}

$* * p<0,01$

\section{Muestras de las ciudades de Buenos Aires y Lincoln}

Se comparan a continuación los resultados de las puntuaciones del item 10 . (Percepción Global de la Calidad de Vida) y del promedio de los 10 items del instrumento (promedio de la Calidad de Vida), entre las muestras obtenidas en las ciudades de Buenos Aires y Lincoln. No se observan diferencias estadísticamente significativas 
con respecto a la Percepción Global de Calidad de Vida. Esto significa que ambas poblaciones perciben un nivel de calidad de vida subjetivamente similar.

En cambio se observan diferencias muy significativas a favor de la ciudad de Lincoln en el promedio de los puntajes de cada uno de los 10 items. Los resultados indican que, considerando la totalidad de las dimensiones que componen el instrumento, los habitantes de Lincoln $(n=48)$ experimentan subjetivamente un mejor nivel de vida que la población de Buenos Aires ( $\mathrm{n}=300$ ), aunque no lo perciben en forma global (ítem 10).

Tabla 5. Comparación Calidad de Vida - BuenosAires-Lincoln

\begin{tabular}{lccccc}
\hline & n & media & desv.típ. & t & \\
\hline Percepción global Buenos Aires & 300 & 7,39 & 1,68 & & \\
Lincoln & 48 & 7,81 & 1,65 & $-1,64$ & NS \\
\hline Promedio CV Buenos Aires & 300 & 7,40 & 1,14 & & \\
Lincoln & 48 & 7,98 & 0,92 & 3,37 & $* *$ \\
\hline
\end{tabular}

$* * p<0,01$

\section{Muestras de las ciudades de Balcarce y Lincoln}

No se observan diferencias estadísticamente significativas entre las ciudades de Balcarce y Lincoln con respecto al ítem 10 - Percepción Global de Calidad de Vida- ni con respecto al promedio de los 10 items del instrumento (promedio CV). En base a los resultados se puede afirmar que ambas poblaciones perciben globalmente y experimentan subjetivamente un nivel de calidad de vida similar con respecto a las 10 dimensiones que componen este instrumento.

Tabla 6. Comparación Calidad de Vida - BuenosAires-Lincoln

\begin{tabular}{lccccc}
\hline & $\mathbf{n}$ & media & desv.típ. & t & \\
\hline Percepción global Balcarce & 50 & 8,02 & 1,30 & & \\
Lincoln & 48 & 7,81 & 1,65 & .69 & NS \\
\hline Promedio CV Balcarce & 50 & 7,96 & 1,67 & & \\
Lincoln & 48 & 7,98 & 0,92 & .09 & NS \\
\hline
\end{tabular}

$p<0,05$

Comparación individual de las medias de cada ítem entre las 3 ciudades

\section{Muestras de Buenos Aires y Balcarce}

La ciudad de Balcarce $(n=50)$ presenta indicadores más elevados que la ciudad de Buenos Aires $(n=300)$ con respecto a la media de los 10 ítems que componen la presente prueba estandarizada. 
Sin embargo se observan diferencias estadísticamente significativas solamente en relación a Funcionamiento Ocupacional (ítem 4) y Apoyo Social-Emocional (ítem 6). Los resultados permiten afirmar que la ciudad de Balcarce experimenta mayor grado de bienestar subjetivo en ambas dimensiones.

Tabla 7. Comparación de ítems 4 y 6 entre Buenos Aires y Balcarce.

\begin{tabular}{lcccc} 
& n & media & des. típ. & t \\
\hline Func. Ocupacional Bs As & 300 & 7,80 & 1,56 & \\
Balcarce & 50 & 9,30 & 9,87 & $2,47^{* *}$ \\
\hline Apoyo Social-Em. Bs As & 300 & 7,61 & 2,20 & \\
Balcarce & 50 & 9,32 & 9,90 & $2,64 * *$ \\
\hline
\end{tabular}

$* * p<0.01$

\section{Muestras de Buenos Aires y Lincoln}

La ciudad de Lincoln $(n=48)$ presenta indicadores más elevados que la ciudad de Buenos Aires $(\mathrm{n}=300)$ con respecto a la media de cada uno de los 10 ítems que componen la presente prueba estandarizada.

Se observan diferencias estadísticamente significativas en relación a 5 ítems: Bienestar Físico (ítem 1), Bienestar Psicológico (ítem 2), Funcionamiento Interpersonal (ítem 5), Apoyo Estatal (ítem 7) y Plenitud Personal (ítem 8). Los resultados permiten afirmar que dicha población experimenta mayor grado de bienestar subjetivo en las cinco variables.

Tabla 8. Comparación de ítems 1,2,5,7 y 8 entre Buenos Aires y Lincoln

\begin{tabular}{lcccc}
\hline & $\mathbf{n}$ & media & des. típ. & t \\
\hline Bienestar Físico Bs As & 300 & 6,98 & 1,78 & \\
Lincoln & 48 & 7,71 & 1,44 & $2,71^{* *}$ \\
\hline Bienestar Emoc. Bs As & 300 & 7,20 & 1,92 & \\
Lincoln & 48 & 8,06 & 1,16 & $3,03 * *$ \\
\hline Func. Interpers. Bs As & 300 & 7,91 & 1,63 & \\
Lincoln & 48 & 8,67 & 1,08 & $3,11^{* *}$ \\
\hline Apoyo Estatal Bs As & 300 & 6,29 & 2,31 & \\
Lincoln & 48 & 7,29 & 2,15 & $2,81^{* *}$ \\
\hline Plenitud Personal Bs As & 300 & 7,44 & 1,85 & \\
Lincoln & 48 & 8,08 & 1,40 & $2,32 *$ \\
\hline$* * p<0.01$ & & & & \\
$*$ p $<0.05$ & & & &
\end{tabular}




\section{Muestras de Balcarce y Lincoln}

La ciudad de Balcarce $(n=50)$ presenta indicadores más elevados que la ciudad de Lincoln $(n=48)$ con respecto a la media de 2 de los 10 ítems que componen la presente prueba estandarizada (Funcionamiento Ocupacional -ítem 4, Apoyo Social Emocional ítem 6), aunque ninguna diferencia adquiere significación estadística.

La ciudad de Lincoln presenta medias superiores con respecto a: Bienestar Físico (ítem 1), Bienestar Psicológico (ítem 2), Cuidado Personal (ítem 3), Funcionamiento Interpersonal (ítem 5), Apoyo Estatal (ítem 7), Plenitud Personal (ítem 8) y Plenitud Espiritual (ítem 9).

Sin embargo se observan diferencias estadísticamente significativas solamente en relación a Bienestar Psicológico (ítem 2). Los resultados permiten afirmar que la población de la ciudad de Lincoln experimenta mayor sensación de bienestar consigo mismo que los habitantes de Balcarce.

Tabla 9. Comparación de ítem 2 entre Balcarce y Lincoln.

\begin{tabular}{lcccc}
\hline & $\mathbf{n}$ & media & des. típ. & t \\
\hline Bienestar Psicológico Balcarce & 507,46 & 1,56 & & \\
\hline Lincoln & 48 & 8,06 & 1,16 & $-2,17^{*}$ \\
\hline$* p<0.05$ & & & & \\
\hline
\end{tabular}

\section{Comentarios y discusión}

En base a los resultados precedentes, podría inferirse que la autopercepción de la calidad de vida no está relacionada con la calidad de vida objetiva.

Según surge del análisis de los indicadores de la calidad de vida objetiva, la ciudad de Buenos Aires presenta mejores condiciones que las ciudades de Balcarce y Lincoln.

Ambas localidades de la provincia de Buenos Aires a su vez presentarían niveles similares de calidad de vida objetiva.

La calidad de vida subjetiva ha sido medida según dos criterios:

A. percepción global de la calidad de vida (ítem 10 del Indice de Calidad de Vida).

B. promedio de los puntajes de los 10 ítems que componen dicho instrumento.

La ciudad de Balcarce presenta un nivel de calidad de vida subjetiva más elevado que la ciudad de Buenos Aires en grado muy significativo, de acuerdo a ambos criterios (A y B).

La ciudad de Lincoln presenta indicadores significativamente más elevados que la ciudad de Buenos Aires, de acuerdo al promedio de los puntajes de los 10 ítems (criterio B). Sin embargo, no se encontraron diferencias entre ambas ciudades con respecto al criterio A (autopercepción global). 
Las ciudades de Balcarce y Lincoln presentan indicadores similares de calidad de vida subjetiva de acuerdo a ambos criterios (A y B).

La comparación de la Percepción Global (ítem 10) por género, indica que en la ciudad de Lincoln las mujeres autoperciben mejor calidad de vida que los hombres.

No se han encontrado diferencias significativas de valoración entre hombres y mujeres en las ciudades de Buenos Aires y Balcarce.

De la comparación de los 9 ítems restantes que componen el Indice de Calidad de Vida, surge que :

- la población de Balcarce percibe mejor nivel de Funcionamiento Ocupacional y de Apoyo Social-Emocional que los habitantes de la ciudad de Buenos Aires.

- en la ciudad de Lincoln la población percibe mejor Bienestar Físico, Bienestar Psicológico, Funcionamiento Interpersonal, Apoyo Estatal y Plenitud Personal que en la ciudad de Buenos Aires.

- los habitantes de Lincoln manifiestan percibir mejor Bienestar Psicológico que la población de Balcarce.

Analizando los resultados entre contextos, se observa que ciertas variables presentes en las condiciones de vida en grandes ciudades tendrían relevancia en la valoración subjetiva de la calidad de vida: inseguridad social, alta densidad demográfica, aislamiento y desorganización.

Un factor importante para comparar condiciones objetivas de vida en una localidad es el nivel de stress que causa la vida actual en grandes ciudades con densidad demográfica elevada, un masa de población que debe recorrer grandes distancias para concurrir a sus destinos laborales, la polución ambiental,-- sonora, atmosférica- , etc.

Las crecientes condiciones de inseguridad y agitación social de la cotidianeidad en la Capital Federal también marcan diferencias.

Según datos registrados por policía, gendarmería y prefectura, por provincia en el 2002, la tasa de delincuencia por cada 10.000 habitantes en Capital Federal es de 663,3. En contraste, la Provincia de Buenos Aires, dicha tasa es de 250,7 delitos.

De acuerdo a información suministrada por la Dirección de Comunicación y Planificación Institucional de la Municipalidad de Balcarce y por la oficina de Prensa de la municipalidad de Lincoln, a marzo del 2004 no se registran delitos contra la libertad de las personas en esas ciudades.

Del análisis de los resultados precedentes surge la siguiente consideración:

Las ciudades de Lincoln y Balcarce no difieren entre sí en los niveles de autopercepción de calidad de vida subjetiva, ni en las condiciones objetivas de vida.

La población de Balcarce percibe globalmente un bienestar más elevado que la ciudad de Buenos Aires en grado muy significativo.

¿Por qué la población de Lincoln percibe globalmente su calidad de vida en forma similar a los habitantes de la ciudad de Buenos Aires? 
¿Podría asignarse a la diferencia de interpretación de este concepto entre las poblaciones?

¿Influyeron las características muestrales (edad, nivel educativo y ocupacional) en la valoración personal de esta variable?

Nuevas mediciones tal vez podrían responder estas cuestiones.

Otra hipótesis podría indicar que los habitantes de la ciudad de Balcarce tienen una mayor conciencia del concepto de calidad de vida a partir de un programa de promoción y prevención de la salud que se desarrolla en dicha ciudad desde hace cuatro años.

En el año 2000 se inició el Proyecto de Demostración Nacional Balcarce-DEMOBALque nuclea a organizaciones no gubernamentales y gubernamentales. La idea del programa es reducir los factores de riesgo de las enfermedades no transmisibles y promover la salud de acuerdo al modelo finlandés del programa de Karelia del Norte.

El Proyecto Karelia tuvo el objetivo de reducir la altísima mortalidad cardiovascular en esa ciudad finlandesa. Se extendió desde 1972 hasta 1992 -hoy se aplica a nivel nacional e internacional- con resultados excelentes. En ese período la mortalidad cardiovascular disminuyó alrededor del $65 \%$ y la del cáncer, un $40 \%$. Reducidas ampliamente las dos causas precedentes, la muerte por todas las causas bajó cerca de un $40 \%$, provocando una mayor expectativa de vida en dicha ciudad finlandesa.

Aplicado en Balcarce, este programa promueve la vida sana, provocando un cambio cultural, con un intenso trabajo psicoeducativo. Entre otras tareas, se forman en criterios de salud a 118 chicos multiplicadores que influirán sobre 900 chicos. Los maestros reciben capacitación especial en la promoción de la salud

Una de las claves es el armado de una trama comunitaria, apoyado en el trabajo voluntario que involucra a personas de todo el espectro etáreo. El programa tiene espacios gratuitos en la televisión de cable y de aire, donde se promocionan estilos saludables de vida.

Aunque el impacto será medible recién en el largo plazo, en el año 2002 la ciudad de Balcarce recibió de la Organización Panamericana de la Salud el $3^{\circ}$ Premio "Municipios Saludables" entre 136 municipios postulados en la categoría Latinoamérica y el Caribe.

Futuras investigaciones podrían profundizar el análisis para determinar el nivel de incidencia del programa mencionado en la autopercepción de la calidad de vida en la ciudad de Balcarce.

De confirmarse dicha hipótesis, sería importante considerar la implementación de programas psicoeducativos y de promoción de la salud en determinadas localidades para intentar amortiguar en parte los efectos que las actuales condiciones socioeconómicas tienen sobre la población. Se considera conveniente aclarar que de ninguna manera se propone sustituir las condiciones externas necesarias para el desarrollo de las personas, sino que el objetivo sería fortalecer al máximo los recursos positivos tanto a nivel social como a nivel individual.

Un efecto secundario de dichos programas es el aumento en las fuentes de apoyo social percibido, al fortalecer la red comunitaria promoviendo vínculos e incrementando la sensación de familiaridad e identidad. Es la información que conduce a los individuos a creer que son cuidados, estimulados y valorados. 
Desde un punto de vista holístico, es un componente de la salud: las relaciones interpersonales positivas presumiblemente protegen a las personas del efecto perjudicial del estrés que traen aparejadas las modalidades vinculares de la vida actual donde se tiende al individualismo y al aislamiento social.

¿ Influyen las circunstancias externas en el bienestar de las personas más que las propias disposiciones individuales? Probablemente estas cuestiones son difíciles de precisar.

Tradicionalmente se mide el bienestar de una población y se trabaja para elevar la calidad de vida de las personas, desde las condiciones externas de vida.

Se considera importante ampliar este enfoque fundamentalmente cuando las condiciones socioeconómicas son adversas y difíciles de cambiar en el corto plazo, como ocurre actualmente en la población argentina.

Frente al panorama actual, la psicología cognitiva moderna, trabajando en aspectos como la evaluación cognitiva, el afrontamiento y la psicoeducación, constituye una alternativa valiosa para mejorar la calidad de vida de las personas.

\section{Referencias bibliograficas}

Antonovsky, R. (1987): Unravelling the mystery of health: How people manage stress and stay well. En Samaniego V. (2002): Tolerancia parental hacia las conductas infantiles: ¿factor de mediación?.Plan de tesis en elaboración para acceder al grado de Doctor en la Universiad de Buenos Aires: subsidio OPS.

Arostegui, (1998): En Gómez Vela N. \& Sabeh, E. (2000): Calidad de Vida. Evolución del concepto y su Influencia en la investigación y la práctica. Integra, 9 (3): 5-13.

Belloch, A., Sandín \& B., Ramos, F.(1995): Manual de Psicopatología-Volumen 2, España, Mac Graw-Hill.

Bulacio, J.M. (2004): Estrés, Ansiedad y Práctica Clínica, Buenos Aires: Acadia.

Bulacio, J.M., Vieyra, M., Daneri, C., Benatuil, D. \& Mongiello, E. (2004): Indice de Calidad de Vida: Validación en una muestra argentina. Alcmeon (en prensa).

Calatayud, M. (1999): Psicología Comunitaria. En Samaniego V.C. (2002): Tolerancia parental hacia las conductas infantiles: ¿factor de mediación?. Plan de tesis en elaboración para acceder al grado de Doctor de la Universidad de Buenos Aires: Subsidio OPS.

Casullo, M.M., et al (2002): Evaluación del bienestar psicológico en Iberoamérica, Buenos Aires: Paidós.

Felce \& Perry (1995): En Gómez Vela N. \& Sabeh E. (2000): Calidad de Vida. Evolución del concepto y su Influencia en la investigación y la práctica. Integra, 9 (3): 5-13. 
Fernández Ballesteros, R (1996): Evaluación en psicología en el campo de la salud: algunos problemas metodológicos. En Casullo, M.M. et al : Evaluación psicológica en el campo de la salud. (pp. 39-89). Buenos Aires, Paidós.

Fischer, C. (1973): On urban alienation and anomia: Powerlessness and social isolation. En Casullo, M.M., et al (2002): Evaluación del bienestar psicológico en Iberoamérica, Buenos Aires: Paidós.

Gómez-Vela, M. \& Sabeh, E.N. (2000): Calidad de Vida. Evolución del concepto y su Influencia en la investigación y la práctica. Integra, 9 (3): 5-13.

Karoly, P.(1985): Measurement Strategies in health psychology. En M.M.Casullo et al (comp): Evaluación psicológica en el campo de la salud (pp. 40). Buenos Aires: Paidós.

Keishman, J. \& Lane, S. (1980): A multivariate analysis of factors affecting perceived life satisfaction and psychological well-being among the elderly. En Casullo, M.M., et al (2002): Evaluación del bienestar psicológico en Iberoamérica, Buenos Aires: Paidós.

Lazarus, R. \& Folkman, S. (1984): Stress, Appraisal and Coping, New York, Springer. Milgram, S. (1970): The experience of living in cities. En Casullo, M.M., et al (2002): Evaluación del bienestar psicológico en Iberoamérica, Buenos Aires: Paidós.

Millon, T. \& Davis, R.D. (1998): Trastornos de Personalidad Más allá del DSM-IV, Barcelona: Masson.

Paglicccia, N.; Apland, L. \& Kazanzian, A. (1995): The impact of professional and personal satisfaction on perceptions of rural and urban: some analytic evidence. En Casullo, M.M., et al (2002): Evaluación del bienestar psicológico e Iberoamérica, Buenos Aires: Paidós.

Ryff, C. \& Keyes, C. (1995): The structure of psychological well-being revisited. En M.M. Casullo, et al (comp): Evaluación del bienestar psicológico en Iberoamérica (pp. 13). Buenos Aires, Paidós, Saforcada, E.(1999): Psicología Sanitaria. Análisis crítico de los sistemas de atención de la salud, Buenos Aires, Paidós.

Samaniego, V.C. (2002): Tolerancia parental hacia las conductas infantiles: ¿factor de mediación?. Plan de tesis en elaboración para acceder al grado de Doctor de la Universidad de Buenos Aires: Subsidio OPS.

Sarason, S. (1974): The psychological sense of community. En Casullo, M. M., et al (2002): Evaluación del bienestar psicológico en Iberoamérica, Buenos Aires: Paidós.

Schalock (1996): En Gómez-Vela, M. \& Sabeh, E.N. (2000): Calidad de Vida. Evolución del concepto y su Influencia en la investigación y la práctica. Integra, 9 (3): 5-13. 
Taylor, S. \& Brown, J. (1988): Illusion and well-being: a social psychological perspective on mental health. En Casullo, M. M., et al (2002): Evaluación del bienestar psicológico en Iberoamérica, Buenos Aires: Paidós.

Usandivaras, C.D. (2004): La salud social es el principal problema de los argentinos. $\mathrm{La}$ Nación (4 de septiembre) pp. 15.

Veenhoven, R. (1988): The utility of happiness, (1991): Is happiness relative?. En M.M.Casullo, et al.(comps). ob. cit. 
Psicodebate 6. Psicología, Cultura y Sociedad 